Far Eastern Entomologist

\begin{tabular}{lll}
\hline Number 445: 7-24 & ISSN 1026-051X (print edition) & January 2022 \\
\hline
\end{tabular}

https://doi.org/10.25221/fee.445.2

http://zoobank.org/References/D853061A-FE2B-4418-AAE7-7019371DBA6E

\title{
LONG-LEGGED FLIES (DIPTERA: DOLICHOPODIDAE) FROM KURILE ISLANDS, WITH DESCRIPTION OF A NEW SPECIES OF THE GENUS SCIAPUS ZELLER, 1842
}

\author{
I. Ya. Grichanov ${ }^{1)}$, O. O. Selivanova ${ }^{2)}$ \\ 1) Institute of Plant Protection, Podbelskogo 3, St. Petersburg-Pushkin, 189620, Russia. E- \\ mail: grichanov@mail.ru \\ 2) Voronezh State University, Universitetskaya Sq. 1, Voronezh, 394006, Russia. E-mail: \\ selivanova-o@list.ru
}

Summary. An annotated list of dolichopodid species recorded from Kurile Archipelago (Russia) is given for the first time. Sciapus basarukini sp. n. from Kunashir Island is described and illustrated. New records for 26 species, including 8 species new for the Kurile Islands, are presented. Long-legged flies are recorded for the first time from the Anuchina, Tanfilyeva, Polonskogo, Urup, Chirpoy, Brat-Chirpoyev, Simushir, Ushishir, Matua, Raikoke, Paramushir, Kharimkotan, Makanrushi, Atlasov, and Onekotan islands. Two species are excluded from the fauna of Kurils. In total, 54 species are recorded from the Kurile Archipelago that apparently makes up $50-60 \%$ of the actual dolichopodid fauna in this region. The trends discovered on the Kurile Islands for plant species, i.e. the landscape diversity and the number of plant species increase from small to large islands, and decrease from southern islands northward, are generally supported by the case of flies of the family Dolichopodidae.

Key words: biodiversity, taxonomy, new species, key, fauna, new records, Russia, East Palaearctic.

И. Я. Гричанов, О. О. Селиванова. Мухи-зеленушки (Diptera: Dolichopodidae) Курильских островов с описанием нового вида рода Sciapus Zeller, 1842 // Дальневосточный энтомолог. 2022. N 445. С. 7-24.

Резюме. Впервые приведен аннотированный список видов долихоподид Курильских островов (Россия). С Кунашира описан новый вид Sciapus basarukini sp. n. Представлены новые сведения о 26 видах, в том числе 8 новых для Курил. Два вида исключены из списка курильских мух-зеленушек. Долихоподиды впервые выявлены с островов Анучина, Танфильева, Полонского, Уруп, Чирпой, Брат Чирпоев, Симушир, Ушишир, Матуа, Райкоке, Парамушир, Харимкотан, Маканруши, Атласов и Онекотан. Всего на Курилах зарегистрировано 54 вида, которые, по-видимому, составляют $40-50 \%$ реальной фауны долихоподид в этом регионе. Установленные для Курильских островов в отношении растений тенденции, т. е. увеличение ландшафтного разнообразия и количества видов растений от малых островов к большим и их уменьшение от южных островов к северу, в целом подтверждаются на примере семейства мух-зеленушек. 


\section{INTRODUCTION}

The collecting of long-legged flies of the family Dolichopodidae in the Kurile Islands started since the 1946 and 1955 expeditions of the Zoological Institute of the Academy of Sciences of the USSR, Leningrad (ZIN). The dolichopodid collections of ZIN, Zoological Museums of Moscow State University (ZMMU), Voronezh State University (VSU), and Federal Scientific Center of the East Asia Terrestrial Biodiversity, Vladivostok (FCBV) were replenished by various expeditions to the Kuriles in 1960-2010. Since 1970, these materials were used in a number of taxonomical and faunistic reviews of dolichopodid fauna of the USSR and Russia published by the famous dipterologist O.P. Negrobov and his disciples. It is worth noting that O.P. Negrobov included in his Palaearctic Catalog many unpublished records of continental species from the Kuriles without label data (Negrobov, 1991). As follows from published later labels for some of these species, they were collected from southern islands (Iturup, Kunashir, Shikotan and Zelyonyi). Negrobov \& Satô (2006) counted 29 species discovered on the Kurile Islands.

Recently, unsorted dolichopodid material collected mainly by the FCBV expeditions to Kuriles was processed by senior author of this paper during his visit to Vladivostok. For this paper, the authors checked originally published localities, if they were referenced, and established the exact locations of some collecting points according to the ZIN and VSU collections. The data of the known dolichopodid fauna of the territory is summarized in the form of an annotated checklist for the first time. The information on the global distribution for each collected species follows Negrobov et al. (2013), Grichanov (2017) and Yang et al. (2018). Remarks are provided where deemed necessary. All specimens are pinned and will be deposited in the collections of ZIN, ZMMU, VSU and FCBV.

Holotype of a new species was studied and photographed with a ZEISS Discovery V-12 stereo microscope and an AxioCam MRc5 camera. Morphological terminology and abbreviations follow Cumming \& Wood (2017) and Grichanov \& Brooks (2017). The lengths of the podomeres are given in millimeters. Body length is measured from the base of the antenna to the tip of abdominal segment 6 . Wing length is measured from the base to the wing apex.

Herein we provide an annotated checklist of Dolichopididae found in the Kurile Archipelago with new records, taxonomic notes, a key to East Palaearctic species of the genus Sciapus Zeller, 1842, and describe one new species of latter genus.

\section{LIST OF SPECIES KNOWN FROM KURILES WITH TAXONOMIC NOTES AND DESCRIPTION OF A NEW SPECIES}

\section{Genus Argyra Macquart, 1834}

\section{Argyra superba Takagi, 1960}

MATERIAL EXAMINED. Shikotan I.: Tserkovnyi Bay, $43^{\circ} 75^{\prime} \mathrm{N}, 146^{\circ} 70^{\prime}$ E, $27-$ 31.VIII 2012, $1 \hat{\jmath}$, Yu. Sundukov.

DISTRIBUTION. Russia (Sakhalin I., Kurile Is.: Kunashir) (Negrobov, 1976b: 47), Japan (Hokkaido, Honshu). Here this species is recorded from Shikotan Island for the first time.

\section{Genus Chrysotimus Loew, 1857}

Chrysotimus spinuliferus Negrobov, 1978

MATERIAL EXAMINED. Kunashir I.: Golovina Volcano, 24.VII 1973, 1§, 1, Kas-

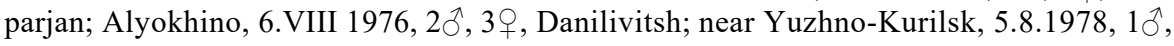


Zlobin; Iturup I.: 18.VIII 1976, O̊, Danilivitsh; Shikotan I.: Krai Sveta Cape, 28.VII 1976,

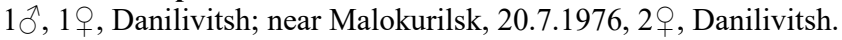

DISTRIBUTION. Russia (Yakutia, Primorsky Krai, Sakhalin I.). It is first record from Kuriles.

\section{Genus Chrysotus Meigen, 1824}

Chrysotus cilitibia Maslova et Negrobov, 2015

DISTRIBUTION. Russia (Sakhalin I., Kurile Is.: Kunashir) (Maslova \& Negrobov, 2015: 201)

\section{Chrysotus saigusai Negrobov, Kumazawa et Tago, 2016}

DISTRIBUTION. Russia (Kurile Is.: Kunashir) (Negrobov et al., 2016: 55), Japan (Honshu).

\section{Chrysotus sp.}

MATERIAL EXAMINED. Zelyonyi I.: 2.VIII 1955, 1우, Violovitsh; Iturup I.: 5 km N Reidovo, Malaise trap, 19.IX 1996, 19, Lelej.

NOTES. The females examined belong to the rich Chrysotus gramineus species group. It is first records of the genus Chrysotus from the Iturup and Zelyonyi islands.

\section{Genus Conchopus Takagi, 1965}

\section{Conchopus borealis Takagi, 1965}

MATERIAL EXAMINED. Kunashir I.: Lagunnoe, 5.VII 1974, 1q, Petrova.

DISTRIBUTION. Russia (Kurile Is.: Kunashir), Japan (Hokkaido); USA (Alabama, California, New Jersey), Peru.

NOTES. Sixteen Conchopus species are known from Japan, but only one species, $C$. borealis, recorded from Hokkaido. The female examined is associated with $C$. borealis because this species was identified previously from males collected from Kunashir (Grichanov, 2002: 90, as Thambemyia borealis).

\section{Genus Condylostylus Bigot, 1859}

\section{Condylostylus nebulosus (Matsumura, 1916)}

MATERIAL EXAMINED. Kunashir I.: Yuzhno-Kurilsk env., 26.VIII 1980, 1ㅇ, Lelei; Alyokhino, 13-16.VIII 1988, 2 ㅇ, Basarukin; 9 km S Yuzhno-Kurilsk, Kislaya River valley,

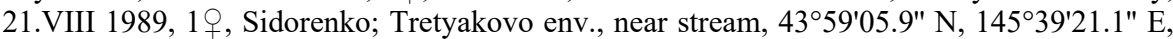

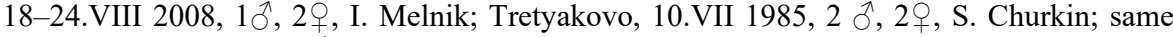
locality, 21.VII 1985, 1 §ै, 2ㅇ, S. Churkin; same locality 21.VII 1984, 1 , , S. Churkin; same locality, 10.VIII 1973, 1 万ิ, Kasparjan; 9 km from Yuzhno-Kurilsk, Mendeleev Volcano, $43.98^{\circ} \mathrm{N} 145.75^{\circ} \mathrm{E}$, 9.VII 1979, 1 ${ }^{\top}$, A. Zinovjev. Japan: [hand-written in Japanese], 14.IV 1928,1 ,, H. Yaku.

DISTRIBUTION. Russia (Kurile Is.: Kunashir) (Negrobov, 1984a: 1113), Japan (Kyushu, Shikoku, Honshu, Hokkaido.), ?Oriental Region. 
NOTES. Four species of Condylostylus are known from Japan, of which two species have yellow mid and hind coxae, i.e., C. nebulosus (type locality in Japan) and C. luteicoxa Parent, 1929 (type locality in India). Bickel (1994) placed C. vigilans Becker, 1922 (described from Taiwan and India) in synonymy to C. nebulosus. The two latter species were not re-described, but C. nebulosus was included in the keys (Bickel, 1994; Yang et al., 2011) with such character as "Mid tarsus [of male] without pale crocheted setae" to distinguish it from C. luteicoxa. However, C. nebulosus was originally described without male secondary sexual characters (Matsumura, 1916), and male mid tarsus of $C$. vigilans was not described by Becker (1922). Males examined from Kunashir have the same rows of crocheted setae on mid tibia and tarsus as those described by Parent (1929) and others for C. luteicoxa, but the colour of the curved setae are black rather than pale. In addition, $C$. luteicoxa was described with reddish-brown antenna, while $C$. nebulosus material from Japan and Kunashir have entirely black antenna. We refer the Kurilian males and females to C. nebulosus. The records of this species outside Japan and Kunashir must be revised.

\section{Genus Dolichopus Latreille, 1796}

\section{Dolichopus agilis Meigen, 1824}

DISTRIBUTION. Europe, Russia (from European part to Far East), Kazakhstan, Mongolia, China (Hebei, Inner Mongolia, Ningxia, Gansu). This species was recorded from Kuriles without definite locality (Negrobov, 1991: 96).

\section{Dolichopus basalis Loew, 1859}

MATERIAL EXAMINED. Shumshu Is.: 24.VII 1978, 10̂, V. Barkalov.

DISTRIBUTION. Russia (from East Siberia to Kamchatka), Korea. It is first record of this species from Kurile Islands.

\section{Dolichopus bigeniculatus Parent, 1926}

MATERIAL EXAMINED. Iturup Is.: "Kuriles, Seseki" [=Iturup Is., Goryachie Klyuchi], fir forest margin, 9.VII 1946, 1§ै, N. Konakov; Kurilsk, 25.VII 1968, 19, V. Richter.

DISTRIBUTION. Russia (Khabarovsky Krai, Primorsky Krai, Kuriles), China (Beijing, Henan, Shaanxi, Shandong, Sichuan, Anhui, Jiangsu, Zhejiang), Japan. This species was recorded from Kuriles without definite locality (Negrobov, 1991: 99).

\section{Dolichopus cuneipennis Parent, 1926}

DISTRIBUTION. Russia (Khabarovsky Krai, Primorsky Krai, Kuriles), China (Beijing, Henan, Shaanxi, Shandong, Anhui, Jiangsu, Sichuan, Zhejiang). This species was recorded from Kuriles without definite locality (Negrobov, 1991: 102).

\section{Dolichopus discifer Stannius, 1831}

MATERIAL EXAMINED. Paramushir I.: Vasiliev Bay, Malaise trap, 16.8.1997, $1 \hat{\delta}$, Lelej, Storozhenko.

DISTRIBUTION. Holarctic species also known from Chukotka, Kamchatka, and Sakhalin Island. It is first record of this species from Kuriles. 


\section{Dolichopus fraterculus Zetterstedt, 1843}

DISTRIBUTION. North Europe (Finland, Lithuania, Norway, Sweden), Russia (from Karelia to Chukotka), USA (Alaska), Canada (Northwest Territories). This species was recorded from Kuriles without definite locality (Negrobov, 1991: 103).

\section{Dolichopus gubernator Mik, 1878}

DISTRIBUTION. Europe (Austria, Estonia, Finland, Latvia, Poland); Russia (from Karelia to Kamchatka, Sakhalin, and Kuriles), Japan. This species was recorded from Kuriles without definite locality (Negrobov, 1991: 104).

\section{Dolichopus impotens Smirnov, 1948}

DISTRIBUTION. Russia (Primorsky Krai, Kuriles). This species was recorded from Kurile Is. without definite locality (Negrobov, 1991: 104).

\section{Dolichopus longicornis Stannius, 1831}

DISTRIBUTION. Holarctic species also known from the Russian Far East (from Chukotka and Kamchatka to Primorsky Krai, Sakhalin I., and Kuriles). This species was recorded from Kuriles without definite locality (Negrobov, 1991: 106).

\section{Dolichopus longicostalis Negrobov et Barkalov, 1978}

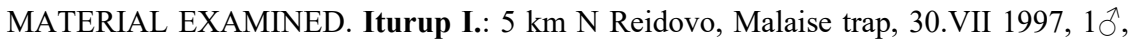
Lelej, Storozhenko; Rybaki, 5 km SW Kurilsk, 22.VI 1968, 1§ิ, 3 ㅇ, V. Rikhter; 25.VI 1968, $2 q$ V. Rikhter; Kunashir I.: Sernovodsk. 26.VIII 1973, 10, Kasparjan; Alyokhino, 29.VII 1973, 1ð, Kasparjan; Lake Lagunnoe, 27.VII 1955, 1 ㅇ Violovich.

DISTRIBUTION. Russia (Sakhalin I). Here this species is recorded from Kurile Ilands for the first time.

\section{Dolichopus migrans Zetterstedt, 1843}

DISTRIBUTION. Europe, Russia (European part, Siberia, Kamchatka, Magadan, Primorsky Krai, Kuriles), Kazakhstan. This species was recorded from Kuriles without definite locality (Negrobov, 1991: 108).

\section{Dolichopus monochaetus Smirnov, 1948}

MATERIAL EXAMINED. Kunashir I.: Golovino, 5VIII 1961, 10ิ, Kupyanskaya (VSU ex coll. ZIN).

DISTRIBUTION. Russia (Primorsky Krai, Kuriles). This species was recorded from Kuriles without definite locality (Negrobov, 1991: 108).

\section{Dolichopus nitidus Fallén, 1823}

MATERIAL EXAMINED. Kunashir I.: Sernovodsk, 27.VIII 1973, 1ठิ, Kasparjan; Golovino, 27.IX 1968, 1ð̊, Gorodkov. 
DISTRIBUTION. Europe, Russia (European part, Siberia, Far East), Kazakhstan, China (Henan, Shanghai), Japan. This species was recorded from Kuriles without definite locality (Negrobov, 1991: 109).

\section{Dolichopus pennatus Meigen, 1824}

DISTRIBUTION. Europe, Russia (European part, Siberia, Far East), Turkey, Mongolia. This species was recorded from Kuriles without definite locality (Negrobov, 1991: 111).

\section{Dolichopus plumipes (Scopoli, 1763)}

MATERIAL EXAMINED. Anuchina I.: 19.VIII 1998, 1§, Lelej, Storozhenko; Atlasova I.: 29.VIII 1976, 1ô, Danilivitsh; Brat Chirpoev I.: 20.VIII 1997, 21 $\hat{\text {, }}$ 5ㅇ, Lelej, Storo-

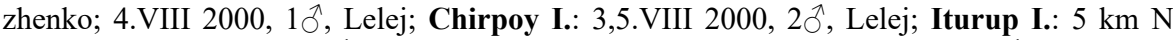
Reydovo, 30.VII 1997, $2 \hat{\jmath}$, Lelej, Storozhenko; Burevestnik, 31.VII 1998, $3 \hat{\jmath}$, 2ᄋ, Lelej,

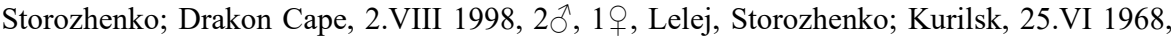

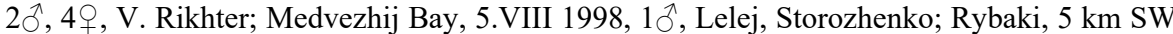
Kurilsk, 22.VI 1968, 1ðึ, V. Rikhter; Kharimkotan I.: 28.VII 2000, 1ð, Lelej, Storozhenko; Kunashir I.: Aliger Lake, 11.VIII 1998, 10, Storozhenko; Alyokhino, 15.VII 1973, 10, Kerzhner; Golovina Vol., 24.VII 1973, 10ิ, Kasparjan; Paltusova Cape, 7.VIII 1955, 19,

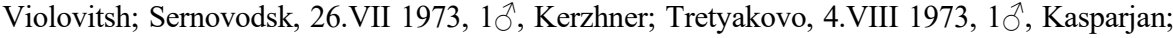
Matua I.: Dvoinaya Bay, 31.VII 2000, $2{ }^{\lambda}$, Lelej, Storozhenko; Onekotan I.: 27.VII 2000, $3 \hat{\jmath}$, Lelej, Storozhenko; Paramushir I.: Krasheninnikova Bay, 14.VIII 1997, 2q, Lelej, Storozhenko; near Severokurilsk, 27.VIII 1976, 4^, 3 , Danilivitsh; Vasilyeva Bay, 16.VIII 19972, 1ठ, 1ㅇ, Lelej, Storozhenko; Polonskogo I.: 21.VIII 1998, 20̂, 2 + , Lelej, Storozhenko; Raikoke I.: eastern coast, 30.VII 2000, $2{ }^{\top}$, Lelej, Storozhenko; Shikotan I.: 2.IX 1948, 1옹 Skufjin; Dmitrieva Bay, 13.VIII 1998, 1ㅇ, Lelej, Storozhenko; Malokurilsk, 18.IX 1968,

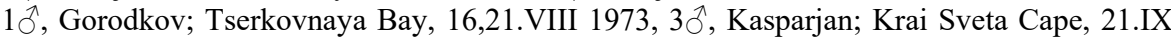

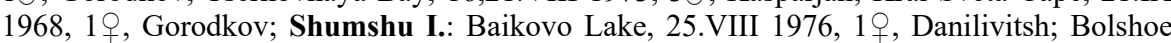
Lake, 9.VIII 1997, 1ð̃, Lelej, Storozhenko; Pochtareva Cape, 7.VIII 1997, $2 \hat{\jmath}, 2$, Lelej,

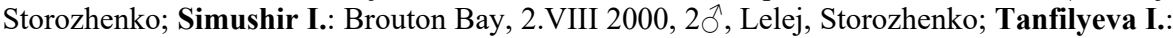

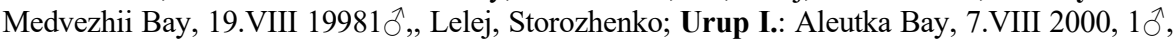
Lelej, Storozhenko; Ushishir I.: Ryponkicha II, 2.VIII 1997, 1ठð, Lelej, Storozhenko; Zelyonyi I.: 2.VIII 1955, 2q, Violovitsh.

DISTRIBUTION. This species is widely distributed in the Palaearctic, Oriental, Nearctic, and Neotropical regions. Here it is recorded from Kurile Islands for the first time.

\section{Dolichopus plumitarsis Fallén, 1823}

DISTRIBUTION. This Holarctic species is known from Europe to Kamchatka, USA (Alaska) and Canada (Ontario). This species was recorded from Kuriles without definite locality (Negrobov, 1991: 112).

\section{Dolichopus ptenopedilus Meuffels, 1982}

MATERIAL EXAMINED. Chirpoy I.: 5.VIII 2000, 1ㅇ, Lelej, Storozhenko; Kharim-

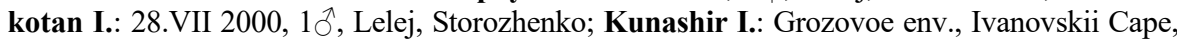
$43^{\circ} 50^{\prime 22} 7^{\prime \prime} \mathrm{N}, 145^{\circ} 24^{\prime} 39.9^{\prime \prime}$ E, 8-15.VIII 2008, 10, I. Melnik; Peschanoe Lake, Danilovo

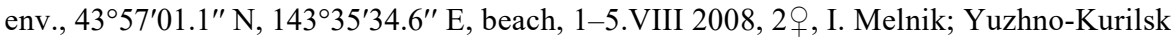


env., 27.VIII 1980, 1§ึ, Storozhenko; Paramushir I.: Vasiliev Bay, Malaise trap, 16.VIII 1997, 1ð̄, Lelej, Storozhenko; 5 km N Severo-Kurilsk, Malaise trap, 14.VIII 1997, 1ð, Lelej, Storozhenko.

DISTRIBUTION. Russia (South Siberia, Far East), Mongolia, Japan. This species was recorded from Kunashur, Shikotan and Iturup islands (Maslova et al., 2012: 76); here it is new for Chirpoy, Kharimkotan and Paramushir islands.

NOTES. Negrobov et al. (2005) used in their key to distinguish $D$. ptenopedilus from $D$. plumitarsis and $D$. simius such variable characters as light colour of lower calypter setae and light at base segment 4 of fore tarsus. However, males of $D$. ptenopedilus are distinct in bearing curved apicoventral seta on segments 1-3 of fore tarsus. The other two species bear straight apicoventral seta on the same segments.

\section{Dolichopus rupestris Haliday, 1833}

DISTRIBUTION. Holarctic species known from Europe to Kamchatka, USA (Alaska) and Canada (Yukon Territory). This species was recorded from Kuriles without definite locality (Negrobov, 1991: 113).

Dolichopus sidorenkoi Negrobov, Maslova et Selivanova, 2011

DISTRIBUTION. Endemic to Shumshu Island (Negrobov et al., 2011).

\section{Dolichopus simius Parent, 1927}

MATERIAL EXAMINED. Shumshu I.: 18-24.VII 1978, 1ठ, V. Barkalov.

DISTRIBUTION. Russia (European part, Siberia, Far East), Mongolia, China (Heilongjiang, Inner Mongolia). This species was recorded from Kuriles without definite locality (Negrobov, 1991: 115).

\section{Dolichopus sinuatus Negrobov et Barkalov, 1978}

MATERIAL EXAMINED. Iturup I.: 7 km W Reydovo, 29.VII 1997, $1 \hat{\jmath}$, Lelej; Kurilsk, 29.VII 1997, 1ðึ, Lelej; Roca Bay, 29.VII 1998, 1ð̂, Lelej; Kunashir I.: Sernovodsk, 26.VIII

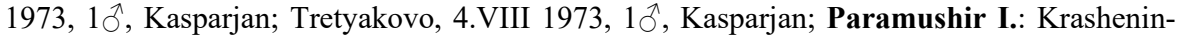
nikova Bay, 14.VIII 1997, 2ð̄, Lelej, Storozhenko; Shikotan I.: Dmitrieva Bay, 13.VIII 1998, 1ð̂, Lelej, Storozhenko.

DISTRIBUTION. Russia (Kuriles, Sakhalin I.). This species was known from Kunashir Island (Negrobov et al., 2010: 523) and here is recorded from Shikotan, Paramushir and Iturup islands for the first time.

\section{Dolichopus stackelbergi Smirnov, 1948}

DISTRIBUTION. This species was described from Primorsky Krai of Russia and was recorded from Kuriles without definite locality (Negrobov, 1991: 116).

\section{Dolichopus storozhenkoi Negrobov, Selivanova et Maslova, 2016}

MATERIAL EXAMINED. Zelyonyi I.: 2.VIII 1955, 1 , , Violovitsh.

DISTRIBUTION. Russia (Sakhalin I., Kurile Is.: Kunashir and Shikotan) (Negrobov et $a l ., 2016: 34)$. It is first record from Zelyonyi Island. 


\section{Dolichopus triangularis Smirnov, 1948}

MATERIAL EXAMINED. Shikotan I.: 2.IX 1948, 10, Skufjin.

DISTRIBUTION. Russia (Primorsky Krai), Japan.). Here this species is recorded from Kuriles for the first time.

\section{Dolichopus varians Smirnov, 1948}

DISTRIBUTION. Russia (Kamchatka, Khabarovsky Krai, Primorsky Krai, Kuriles), Korea. This species was recorded from Kuriles without definite locality (Negrobov, 1991: 118).

\section{Dolichopus verae Negrobov, 1977}

MATERIAL EXAMINED. Iturup I.: 5 km N Reydovo, 30.VII 1997, 20̂, Lelej, Storozhenko; Kunashir I.: Sernovodsk, 26.VIII 1973, 1ð̂, Kasparjan; Shikotan I.: Malokurilsk, hillock 412, 18.VIII 1971, Ô, Nartshuk.

DISTRIBUTION. Russia (Kuriles, Sakhalin I.). This species was described from Iturup (Negrobov, 1977a: 100); present data are first records from Kunashir, Shikotan and Iturup islands.

\section{Dolichopus violovitshi Negrobov, 1977}

MATERIAL EXAMINED. Kunashir I.: 9 km S Yuzhno-Kurilsk, River valley, 27.VII

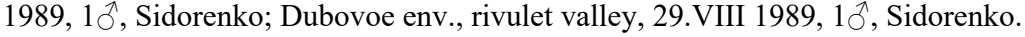

DISTRIBUTION. Russia (Kamchatka, Sakhalin, Kuriles). This species was described from Iturup Island (Negrobov, 1977a: 103); here we found it in Kunashir Island.

\section{Dolichopus xanthopyga Stackelberg, 1930}

DISTRIBUTION. Russia (Khabarovsky Kray, Primorsky Krai, Sakhalin, Kuriles), China (Heilongjiang). This species was recorded from Kuriles without definite locality (Negrobov, 1991: 118).

\section{Genus Gymnopternus Loew, 1857}

\section{Gymnopternus daubichensis (Stackelberg, 1933)}

MATERIAL EXAMINED. Kunashir I.: Mendeleevo, 3.VIII 1975, 1ð̋, Berezantsev.

DISTRIBUTION. Russia (Primorsky Krai, Kuriles). This species was recorded from Zelyonyi Island as Hercostomus daubichensis (Negrobov, 1991: 85); here we found it in Kunashir Island.

\section{Gymnopternus pseudoceler (Stackelberg, 1933)}

MATERIAL EXAMINED. Iturup I.: 5 km N Reidovo, Malaise trap, 30.VII 1997, 1 ${ }^{\Uparrow}$, Lelej, Storozhenko.

DISTRIBUTION. Russia (Primorsky Krai, Kuriles). This species was recorded from Iturup and Zelyonyi islands as Hercostomus pseudoceler (Negrobov \& Rodionova, 2004: 203). 


\section{Gymnopternus ussurianus (Stackelberg, 1933)}

MATERIAL EXAMINED. Kunashir I.: Sernovodsk, 27.VII and 26.VIII 1973, 30 , Kasparjan; Golovino, 27.IX 1968, 10, Gorodkov; Shikotan I.: near Malokurilsk, 20.VIII

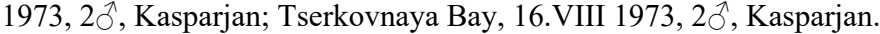

DISTRIBUTION. Russia (Amurskaya oblast, Khabarovsky Krai, Primorsky Krai, Kuriles), Japan. This species was recorded from Kunashir as Hercostomus ussurianus (Negrobov \& Rodionova, 2004: 203). Here it is recorded from Shikotan Island for the first time.

\section{Genus Hercostomus Loew, 1857}

\section{Hercostomus rivulorum Stackelberg, 1934}

DISTRIBUTION. Russia (Primorsky Krai, Sakhalin, Kuriles). Species was recorded from Kunashir Island (Nechai, 2011: 108).

\section{Hercostomus rohdendorfi Stackelberg, 1934}

DISTRIBUTION. Russia (Yakutia, Khabarovsky Krai, Primorsky Krai, Kuriles). This species was recorded from Shikotan Island (Nechai, 2011: 109).

Genus Medetera Fischer von Waldheim, 1819

Medetera alexandri Negrobov, 1979

DISTRIBUTION. Endemic to Kunashir Island (Negrobov, 1979: 656).

Medetera fasciata Frey, 1915

DISTRIBUTION. Europe, Russia. This species was recorded from Kuriles without definite locality (Negrobov, 1991: 125).

\section{Genus Mesorhaga Schiener, 1868}

Mesorhaga dimi Negrobov, 1984

DISTRIBUTION. Russia (Kunashir) (Negrobov, 1984b: 32), Korea.

Genus Microphorella Becker, 1909

Microphorella emiliae Shamshev, 2004

DISTRIBUTION. Endemic to Kunashir Island (Shamshev, 2004: 528).

\section{Genus Neurigona Rondani, 1856}

\section{Neurigona anomaloptera Negrobov, 1987}

DISTRIBUTION. Endemic to Kunashir Island (Negrobov, 1987: 415). 


\section{Neurigona flavella Negrobov, 1987}

DISTRIBUTION. Russia (Primorsky Krai, Kuriles). This species was recorded from Kunashir Island (Negrobov, 1987: 409).

Neurigona kasparyani Negrobov, 1987

DISTRIBUTION. Russia (Sakhalin and Kunashir islands), Japan. This species was described from Kunashir Island (Alekhino) (Negrobov, 1987: 412).

\section{Neurigona micropyga Negrobov, 1987}

DISTRIBUTION. Russia (Khabarovsky Krai, Primorsky Krai, Kuriles), China (Henan), Japan. Kunashir is the type locality of this species (Negrobov, 1987: 413).

\section{Genus Rhaphium Meigen, 1803}

\section{Rhaphium sp. 1}

MATERIAL EXAMINED. Paramushir I.: Vasiliev Bay, Malaise trap, 15.VIII 1997, 1 , , Lelej, Storozhenko.

NOTES. The female examined belongs to a group of species from the former genus Porphyrops Meigen, 1824 (sensu Becker, 1918). This is first record of the genus Rhaphium from Paramushir Island.

\section{Rhaphium sp. 2}

MATERIAL EXAMINED. Urup I.: Chernoburka Bay, 9.VIII 2000, 19, Lelej, Storozhenko.

NOTES. The female examined belongs to a group of species from the former genus Xiphandrium Loew, 1857 (with white bristle on hind coxa). This is first record of the genus Rhaphium from Urup Island.

\section{Rhaphium gussakovskii Stackelberg et Negrobov, 1976}

DISTRIBUTION. Russia (Primorsky Krai, Kuriles). This species was recorded from Iturup Island (Negrobov, 1976a: 860).

\section{Rhaphium richterae Negrobov, 1977}

DISTRIBUTION. Endemic to Kunashir Island (Negrobov, 1977b: 46; Kleshchevnikova \& Negrobov, 2017: 54)

\section{Genus Syntormon Loew, 1857}

\section{Syntormon violovitshi Negrobov, 1975}

MATERIAL EXAMINED. Iturup I.: 20.IX 1967, 1ð, Sytshevskaya; 5 km N Reidovo, 30.VII 1997, 1일. Lelej, Storozhenko; same locality, Malaise trap, 30.VII 1997, 1 9 , Lelej, Storozhenko; Kurilsk, 25.VI 1968, 3 q, V. Rikhter; Rybaki, 5 km SW Kurilsk, 22.VI 1968, 
19, V. Rikhter; Kunashir I.: Sernovodsk, 14.VII 1973, 20ิ, Logvinovskii; Sernovodsk, 28.VI 1973, 19, Kerzhner; Makanrushi I.: Malaise trap, 18.VIII 1997, 19, Lelej, Storozhenko; Urup I.: Chernoburka Bay, 7.VIII 2000, 1ð^, Lelej, Storozhenko.

DISTRIBUTION. Russia (Kamchatka, Sakhalin), Korea, Japan. Here this species is recorded from Kuriles for the first time.

\section{Genus Sciapus Zeller, 1842}

NOTES. The Palaearctic species of Sciapus were revised recently (Grichanov \& Negrobov, 2014). Later Sciapus negrobovi Naglis et Bartak, 2015 was described from West Palaearctic (Portugal). The genus contains about 80 species worldwide, including 69 recognized species from the Palaearctic, eight from the Nearctic, two from Oriental regions and three species from Afrotropics. Sciapus is not species-rich in East Palaearctic, containing here eight species with only one Trans-Palaearctic species, Sciapus nervosus (Lehmann, 1822). The latter is the only representative of the genus in Japan. Below a new species of the genus from Kunashir is described, and an identification key to males of East Palaearctic species is provided.

\section{Key to East Palaearctic species of the genus Sciapus (males)}

1. Tarsi with one or more segments enlarged (often slightly) 2

- All tarsi simple, at most some segments with erect setulae, or elongated, or sometimes 5 th segment flattened dorsoventrally and slightly broadened

2. Mid tarsus with 3rd segment not enlarged, and 4th segment slightly enlarged; fore tarsus simple; body length 5.5-5.8 $\mathrm{mm}$ (Primorye)

S. dytei Negrobov, Maslova et Selivanova, 2012

- Mid tarsus simple; fore tarsus with 4th segment bearing dorsal lobe

... 3

3. Acrostichals present; wing vein $d m-m$ straight; body length 7.9-8.1 mm (Primorye, Sakhalin)

S. paradoxus Negrobov et Shamshev, 1986

- Acrostichals absent; wing vein $d m-m$ strongly convex

4. Epandrial lobe much longer than epandrium, bearing long hairs; antennal pedicel with pale bristles; hind basitarsus much longer than next segment; body length 5.0-8.0 mm (TransPalaearctic) S. nervosus (Lehmann, 1822)

- Epandrial lobe shorter than epandrium, sparsely ciliated; antennal pedicel with dark bristles; body length $6.0 \mathrm{~mm}$ (Amurian Region, Primorye) S. roderi Parent, 1929

5. Frons, mesonotum and abdomen entirely shining green, only very feebly dusted; cerci mostly fused, each cercus with long free distal projection; organ X (ventral cercal projection) reduced

- Frons, thorax and abdomen not shining, densely pollinose, sometimes with shining spots or stripes; cerci fused, organ X large

6. Distoventral projection of cercus with short simple setae; surstylus with simple setae at apex; body length 4.7-4.9 mm (Primorye)

S. incognitus Negrobov et Shamshev, 1986

- Distoventral projection of cercus with ventral fringe of strong flattened bristles and 1 long undulate flattened apical seta; ventral process of surstylus with 2 long undulate flattened apical setae; body length $5.0 \mathrm{~mm}$ (Kunashir) S. basarukini sp. n.

7. Abdomen entirely dark, rarely with yellow-brown spots laterally at base; body length 4.8 mm (Buryatia, Chita, Irkutsk, Primorye) ......... S. sibiricus Negrobov et Shamshev, 1986

- Abdominal segments 1-4 at least partly yellow 
8. Lobes of organ X bifurcate, with short apical setae; body length $5.0 \mathrm{~mm}$ (Gansu, Inner Mongolia) ..... S. flexicornis Parent, 1944

- Lobes of organ X fused to apex, thin and beaked at apex, with curved dorsally distal process; with uninterrupted row of ventral setae decreasing in length towards apex; fore and mid tarsi covered with erect setulae; body length $3.6 \mathrm{~mm}$ (Yakutia)

S. vladimiri Grichanov et Negrobov, 2014

Sciapus basarukini Grichanov et Selivanova, sp. $\mathbf{n}$.

http://zoobank.org/References/D853061A-FE2B-4418-AAE7-7019371DBA6E

Figs 1-6

TYPE MATERIAL. Holotype - $\hat{\jmath}$, Russia: Kuriles, Kunashir Island, Alekhino, 13-16.VIII 1988, leg. A.M. Basarukin (ZIN).

DESCRIPTION. MALE. Head: as wide as high; frons shining bluish green, weakly grey pollinose; 1 short front vertical bristle, half as long as postvertical, located close to latter bristle; pair of strong black oculars; 1 long black postvertical; face somewhat bulging, weakly shining under antennae, white pollinose, under antennae 4 times wider than postpedicel height; clypeus densely white pollinose, adjacent to eyes; proboscis brown; palpus light brown, with black seta and white hairs; antenna yellow; pedicel with short setae; postpedicel about as long as high, haired; stylus brown-black, dorsal, shortly haired, 2.5 times longer than antennomeres combined; length (mm) of scape, pedicel, postpedicel, stylus (segments 1 and 2), 0.08/0.06/ 0.07/0.06/0.67; upper 3-5 postocular setae black, lateral and lower postoculars white.

Thorax: mesonotum shining bluish green, with black setae; pleura black-green, grey pollinose; five dorsocentrals decreasing in length anteriorly; acrostichals absent; scutellum with 2 strong setae (broken).

Legs: with fore tarsomeres 3-5, mid and hind tarsi broken; coxae and other podomeres yellow; coxae with yellow setae and hairs; fore coxa with hairs anteriorly and 3 apical setae; fore femur with 1-2 ventral light setae at base, at most as long as femur height; fore tibia and basitarsus without distinct setae; mid femur with minute ventral white hairs, with anterior and posterior rows of elongate semierect setulae on distal half; no strong anterior preapical seta; mid tibia with 1 anterodorsal seta at base, with rows of erect setulae on distal half; hind femur with strong anterior preapical seta (broken); hind tibia with 4-5 dorsal setae. Fore coxa, femur, tibia and tarsomere (first to second) length ratio $(\mathrm{mm}): 0.75 / 1.43 / 1.53 / 1.46 / 0.51$; mid femur and tibia length ratio (mm): 1.4/2.84; hind femur and tibia length ratio (mm): 1.93/2.75.

Wing: hyaline; costa straight; ratio of part of costa between $\mathrm{R}_{2+3}$ and $\mathrm{R}_{4+5}$ to that between $\mathrm{R}_{4+5}$ and $\mathrm{M}_{1}: 0.52 / 0.1$; crossvein $d m-m$ straight; $\mathrm{M}_{2}$ foldlike, $\mathrm{M}_{4}$ well developed; anal lobe narrow; anal angle right; length ratio of $d m-m$ to distal part of $\mathrm{M}_{1+2}$ (fork-handle) to distal part of $\mathrm{M}_{4}:$ 0.48/0.37/0.61 (in mm); lower calypter with yellow cilia; halter yellow.

Abdomen: shining greenish-blue, black along sutures, with mainly light setae; sterna dark; hypopygium black with light brown appendages; segment 7 short; segment 8 large, covered with light hairs.

Hypopygium with long simple phallosome; epandrial lobe finger-like, with 2 long setae at apex; surstylus as long as cercus, with curved dorsal and simple ventral short processes at apex; ventral process of surstylus with short simple and 2 long undulate flattened apical setae; cerci fused at base, with relatively long light hairs and black setae on basal part, with reduced basoventral projection (organ X) covered with short setae; distoventral projection of cercus free, with ventral fringe of strong flattened bristles and 1 long undulate flattened apical seta.

MEASUREMENTS (mm). Body length: 5.0, wing length: 4.3, wing width: 1.4, antenna length: 0.9 . 
FEMALE. Unknown.

DIAGNOSIS. The new species keys to Sciapus incognitus Negrobov et Shamshev, 1986 (Grichanov, Negrobov, 2014) described from Primorsky Krai and is very similar to the latter (Negrobov, Shamshev, 1986). Sciapus basarukini sp. n. differs in distoventral projection of
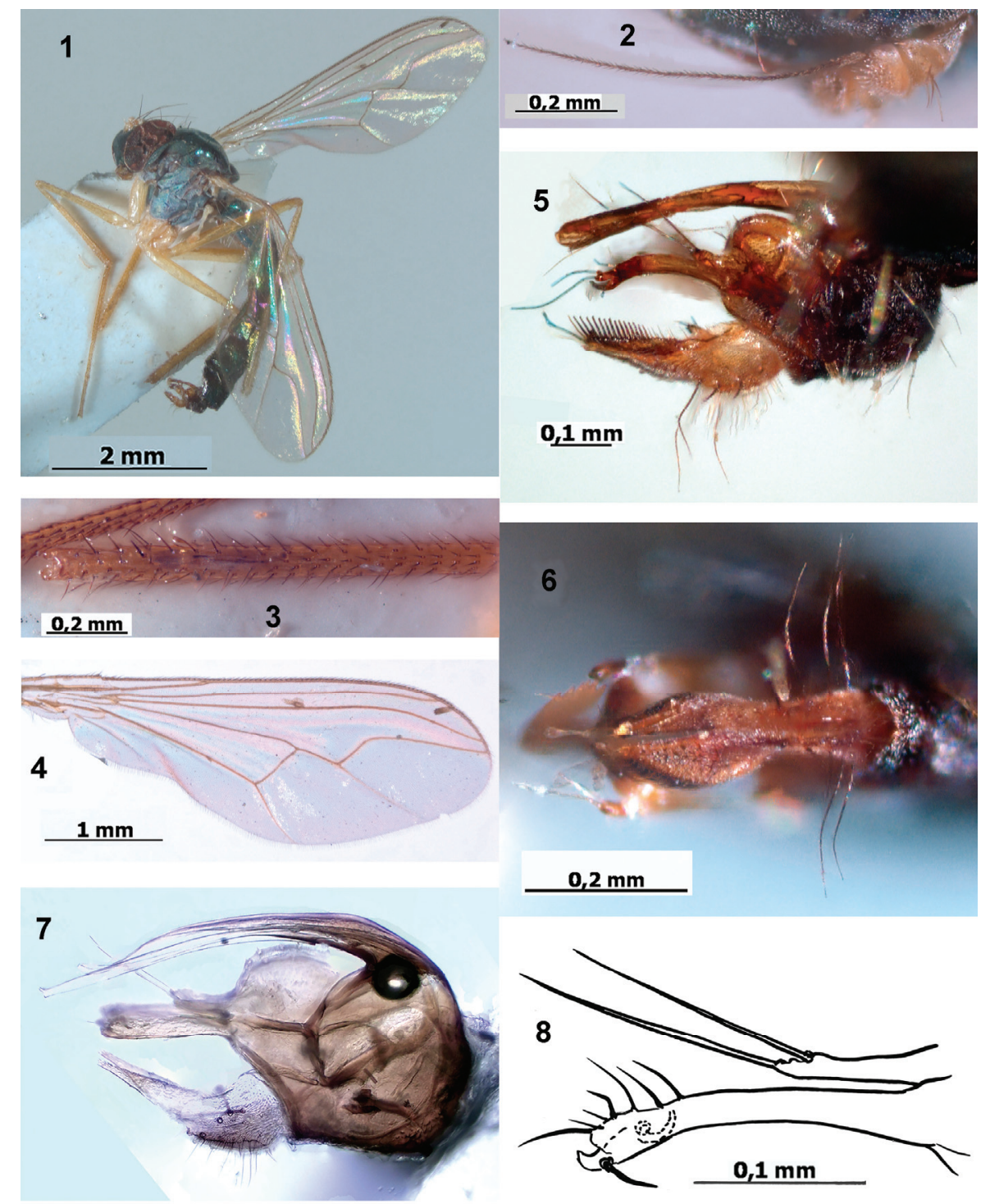

Figs 1-8. Sciapus spp.: 1-6 - S. basarukini sp. n., holotype, male: 1 - habitus; 2 - antenna; 3 - mid tibia, distal half; 4 - wing; 5 - hypopygium, lateral view, dry; 6 - cerci, dorsal view, dry; 7, $8-S$. incognitus Negrobov et Shamshev, 1986 (paratype): 7 - hypopygium, lateral view, after macertion; 8 - surstylus, lateral view. 
cercus with ventral fringe of strong flattened bristles and presence of long undulate flattened apical seta; ventral process of surstylus with two long undulate flattened apical setae (Fig. 5). Sciapus incognitus male has only short simple setae on apex of cercus and surstylus (Figs 7, 8). Mid tarsus of this species bears a row of anterodorsal setae. Mid tarsi of $S$. basarukini sp. n. holotype are broken, but its mid tibia is covered with rows of erect setulae on distal half (Fig. 3); we suggest that its mid tarsus is also covered with erect setulae, such as mid tarsi of similar European S. longulus (Fallén, 1823), S. euchromus (Loew, 1857) and S. laetus (Meigen, 1838) males (Grichanov, Negrobov, 2014).

ETYMOLOGY. The name of the new species is dedicated to the collector of the holotype, Russian zoologist Anatolii Mikhailovich Basarukin (1952-1995).

\section{SPECIES EXCLUDED FROM THE FAUNA OF KURILE ISLANDS}

\section{Dolichopus setimanus Smirnov, 1948}

NOTES. Describing their new species, Dolichopus storozhenkoi (Negrobov et al., 2016), the authors used the material collected from Kuriles (and partly from Sakhalin) and listed under D. setimanus (Selivanova et al., 2010). So, the latter species is excluded from the list of recorded from Kuriles Dolichopodidae.

\section{Rhaphium albifrons Zetterstedt, 1843}

NOTES. This species was recorded from "Kuril Islands: Moneron Island" (Negrobov et al., 2020: 890). Moneron is a small island near Sakhalin Island in the Tartar Strait. It does not belong to the Kurile Archipelago. Therefore, Rhaphium albifrons must be excluded from the fauna of Kuriles.

\section{DISCUSSION}

Presently, more than 650 species of Dolichopodidae are known from the continental Chinese and Russian regions belonging to the East Palaearctic, including 93 species found on Kamchatka (Grichanov et al., 2021). The Japanese fauna of long-legged flies numbers 139 identified species and 90 unidentified morphospecies (Kumazawa, 2017). Negrobov \& Satô (2006) counted 48 species discovered on the Sakhalin.

The present research features new records for 26 species, including 8 species new for the Kurile Islands. Long-legged flies are recorded for the first time from the Anuchina, Tanfilyeva, Polonskogo, Urup, Chirpoy, Brat-Chirpoyev, Simushir, Ushishir, Matua, Raikoke, Paramushir, Kharimkotan, Makanrushi, Atlasov and Onekotan islands. In total, 54 species are recorded here that apparently make up 50-60\% of the actual dolichopodid fauna of the Kurile Archipelago.

According with Ganzey (2010), the Kuriles are divided into Northern-Middle and Southern Kurile physiographic provinces. The Southern Kurile province includes Lesser Kurile, Southern Kurile and Urup-Chirpoy physiographic circuits. The Northern-Middle Kurile province includes Northern and Middle Kurile physiographic circuits. Ganzey (2010) referred each big island and group of small islands to separate physiographic district with specific heat supply, soil type and plant community diversity. It was noted (Ganzey \& Ivanov, 2012) that the landscape diversity and the number of plant species increase from small to large islands, and decrease from southern islands northward. Biogeographically, the Lesser Kuriles and Southern Kuriles share with Sakhalin and Hokkaido such biome as deciduous forests, and the other islands share with Kamchatka such biome as meadows and sparse forests (Olson et al., 2001). 
Our data provided in this paper show that the southernmost Kunashir Island is the most species-rich regarding the dolichopodids, with 27 known species including southern Palaearctic elements: Conchopus borealis, Gymnopternus daubichensis, Hercostomus rivulorum, Neurigona flavella, N. kasparyani, N. micropyga, and endemics of this Island: Mesorhaga dimi, Microphorella emiliae, Neurigona anomaloptera, Rhaphium richterae, and Sciapus basarukini sp. n. The Lesser Kuriles (Shikotan, Tanfilyeva, Anuchina, Polonskogo and Zelyonyi islands) are inhabited by 12 species, and the Iturup Island - by 12 species including southern Palaearctic elements: Chrysotimus spinuliferus, Dolichopus longicostalis, D. sinuatus, D. storozhenkoi, D. triangularis, D. verae, Gymnopternus pseudoceler, and Rhaphium gussakovskii. In addition, at least some of the 13 species mentioned by Negrobov (1991) as collected from the Kurile Islands inhabit the Lesser Kurile and Southern Kurile physiographic circuits. In total, at least 40 species inhabit these circuits.

Three widely distributed species and one morphospecies are known from the UrupChirpoy physiographic circuit (sensu Ganzey, 2010), including one Holarctic (Dolichopus plumipes) and two East Palearctic species. Only D. plumipes was collected from the Middle Kurile physiographic circuit (Matua, Raikoke, Simushir, and Ushishir islands). Eight widely distributed species and one morphospecies are found in the Northern Kurile physiographic circuit (Makanrushi, Kharimkotan, Onekotan, Atlasova, Paramushir, and Shumshu islands). Dolichopus sidorenkoi is at present endemic of the Shumshu Island, D. sinuatus is known from Kuriles and Sakhalin, and the other identified species are widespread, known also from the nearby Kamchatka Peninsula. Three species are East Palearctic, and two species are Holarctic in their distribution (Dolichopus discifer and D. plumipes). The Northern Kurile Dolichopus simius is reported from Palearctic China, Mongolia, Siberia and Far Eastern Russia, but also from Bashkortostan, Moscow and Sverdlovsk regions; if not a synonym of the Holarctic D. plumitarsis, it should be considered an overlooked species in the European fauna until the material from various parts of its area is directly compared with $D$. plumitarsis.

So, the trends discovered on the Kurile Islands for plant species (Ganzey \& Ivanov, 2012) are generally supported by the case of fly family Dolichopodidae.

\section{ACKNOWELDGEMENTS}

The authors are sincerely grateful to Prof. Arkadij Lelej (FCBV), Drs N.E. Vikhrev and A.L. Ozerov (ZMUM), and Dr. Igor Shamshev (ZIN) for their kindness in providing specimens for study. Dr. S.Yu. Storozhenko (FCBV) kindly commented on earlier draft of the manuscript. The work was funded by RFBR and NSFC according to research project No. 20 54-53005. The identification of the new material from the FCBV collection was supported by All-Russian Institute of Plant Protection project No. 0665-2019-0014.

\section{REFERENCES}

Becker, T. 1918. Dipterologische Studien. Dolichopodidae. A. Paläarktischen Region. Nova Acta Academiae Caesareae Leopodinisch-Carolinae Germanicae Naturae Curiosorum, 103: 203-315.

Becker, T. 1922. Dipterologische Studien. Dolichopodidae der Indo-Australischen Region. Capita Zoologica, 1(4): 1-247.

Bickel, D.J. 1994. The Australian Sciapodinae (Diptera: Dolichopodidae), with a review of the Oriental and Australasian faunas, and a world conspectus of the subfamily. Records of the Australian Museum, Supplement, 21: 1-394. DOI: 10.3853/j.0812-7387.21.1994.50 
Cumming, J.M. \& Wood, D.M. 2017. 3. Adult morphology and terminology. P. 89-134. In: Kirk-Spriggs, A.H. \& Sinclair, B.J. (Eds.). Manual of Afrotropical Diptera. Volume 1. Introductory chapters and keys to Diptera families. Suricata 4. Pretoria, SANBI Graphics \& Editing.

Ganzey, K.S. 2010. Landscapes and physiographic zoning of the Kuril Islands. Vladivostok, Dalnauka, 214 p. [in Russian].

Ganzey, K.S. \& Ivanov, A.N. 2012. Landscape diversity of the Kuril Islands. Geography and natural resources, 2: 87-94 [in Russian].

Grichanov, I.Ya. 2002. New and rare Dolichopodidae (Diptera) in the fauna of Russia. P. 90-91. In: Tezisy XII s'ezda Russkogo Entomologicheskogo obshchestva. SanktPeterburg, 19-24 avgusta [Proceedings of the 12th Congress of Russian Entomological Society. Saint Petersburg, 19-24 August 2002]. Saint Petersburg, Russian Entomological Society Publ. [In Russian]

Grichanov, I.Ya. 2021. Alphabetic list of generic and specific names of predatory flies of the epifamily Dolichopodoidae (Diptera). (Online version). Saint Petersburg, All-Russian Research Institute of Plant Protection Publ. Available from: http:/grichanov.aiq.ru/ genera3.htm (Accessed 1 November 2021).

Grichanov, I.Ya. \& Brooks, S.E. 2017. 56. Dolichopodidae (long-legged dance flies). P. 1265-1320. In: Kirk-Spriggs, A.H. \& Sinclair, B.J. (Eds.). Manual of Afrotropical Diptera, Volume 2. Nematocerous Diptera and Lower Brachycera. Suricata 5. SANBI Graphics \& Editing, Pretoria.

Grichanov, I.Ya., Chursina, M.A. \& Wang M. 2021. Detection of biodiversity local centers and gradients of change of Dolichopodidae (Diptera) in East Asia. Journal of Insect Biodiversity, 28(1): 13-34. DOI: 10.12976/jib/2021.28.1.2

Grichanov, I.Ya. \& Negrobov, O.P. 2014. Palaearctic species of the genus Sciapus Zeller (Diptera: Dolichopodidae). Saint Petersburg, All-Russian Research Institute of Plant Protection Publ., 84 p. Available from: https://archive.org/details/GrichanovNegrobov2014Sciapus (Accessed 1 November 2021).

Kumazawa, T. 2017. Information on Diptera of Japan. A checklist of Dolichopodidae (Diptera) of Japan. (Online version). Available from: https://diptera-bio.jimdofree.com/ (Accessed 1 November 2021).

Maslova, O.O. \& Negrobov, O.P. 2015. New species of Chrysotus Meigen, 1824 (Diptera: Dolichopodidae) from Sakhalin and Kuril Islands (Russia). Caucasian Entomological Bulletin, 11(1): 201-203. [In Russian]

Maslova, O.O., Negrobov, O.P. \& Selivanova, O.V. 2012. The first records of Dolichopus ptenopedilus (Dolichopodidae, Diptera) from Russia and Mongolia with description of its female. Amurian Zoological Journal, 4(1): 76-78.

Nechai, N.A. 2011. Taxonomy, fauna, and zoogeography of the genus Hercostomus Loew (Dolichopodidae, Diptera) of the Palaearctic. PhD Thesis (MS). Saint Petersburg, 178 pp. [In Russian]

Negrobov, O.P. 1976a. New species of the genus Rhaphium (Dolichopodidae, Diptera) in Siberia and the Far East. Zoologicheskii Zhurnal, 55(6): 860-865. [In Russian]

Negrobov, O.P. 1976b. New and little-known species of family Dolichopodidae of USSR adjacent territories fauna. Nauchnye doklady vysshei shkoly, Biologicheskie nauki, 8: 4550. [In Russian]

Negrobov, O.P. 1977a. New species of the genus Dolichopus Latreille (Dipt. Dolichopodidae) from Siberia and Far East. In: Cherepanov A.I. (ed.). Taxa of Siberian fauna. New and little-known species of Siberian fauna. Novosibisk, 11: 92-104. [In Russian] 
Negrobov, O.P. 1977b. New species of genus Rhaphium (Diptera, Dolichopodidae) of USSR fauna. Vestnik zoologii, 1: 45-49. [In Russian]

Negrobov, O.P. 1979. Diptera of family Dolichopodidae (Diptera) of the USSR fauna. I. Subfamilies Dolichopodinae and Medeterinae. Entomologicheskoe Obozrenie, 58(3): 646-657. [In Russian]

Negrobov, O.P. 1984a. Genera new for Palearctic and USSR fauna of family Dolichopodidae (Diptera). Zoologicheskii Zhurnal, 63(7): 1111-1115. [In Russian]

Negrobov, O.P. 1984b. Species of the genus Mesorhaga Schiner (Dolichopodidae) in Palearctic fauna. Nauchnye doklady vysshei shkoly, Biologicheskie nauki, 8: 31-35. [In Russian]

Negrobov, O.P. 1987. New Palearctic species of genus Neurigona Rond. (Diptera, Dolichopodidae). Entomologicheskoe Obozrenie, 66(2): 406-415. [In Russian]

Negrobov, O.P. 1991. Family Dolichopodidae. P. 11-139. In: A. Soos, L. Papp (eds.). Catalogue of Palaearctic Diptera. Vol. 7: Dolichopodidae - Platypezidae. Budapest, Akademiai Kiado Publ. DOI: 10.1016/B978-0-444-98732-0.50008-9

Negrobov, O.P., Barkalov, A.V. \& Selivanova, O.V. 2010. New data on the fauna of the family Dolichopodidae (Diptera) from Russia, with a description of a new species of the genus Argyra Mcq. Euroasian Entomological Journal, 9(3): 522-524.

Negrobov, O.P., Kumazawa, T., Tago, T. \& Fursov, V.N. 2016. Species of the genus Chrysotus Meigen, 1824 (Diptera: Dolichopodidae) from Japan, with descriptions of two new species. European Journal of Taxonomy, 197: 1-15.

Negrobov, O.P., Maslova, O.O. \& Selivanova, O.V. 2011. New and little-known species of the genus Dolichopus Latreille, 1796 (Diptera: Dolichopodidae) from Russia. Far Eastern Entomologist, 232: 11-16.

Negrobov, O.P., Oboňa, J., Manko, P. \& Maslova, O.O. 2020. New faunistics notes on the fauna and variability of Rhaphium albifrons Zetterstedt, 1843 (Dolichopodidae: Diptera). P. 89-90. In: Prostranstvenno-vremennye aspekty funktsionirovaniya biosistem Sbornik materialov XVI Mezhdunarodnoi nauchnoi ekologicheskoi konferentsii, posvyashchennoi pamyati Aleksandra Vladimirovicha Prisnogo. Belgorod, BelGU Publ.

Negrobov, O.P. \& Rodionova, S.Y. 2004. New data on fauna of subfamily Dolichopodinae (Dolichopodidae, Diptera) in Russia and neighbouring territories (genus Hercostomus Lw.). An International Journal of Dipterological Research, 15(3): 201-204.

Negrobov, O.P., Rodionova, S.Yu., Maslova, O.O. \& Selivanova, O.V. 2005. Key to the males of the Palaearctic species of the genus Dolichopus Latr. (Diptera: Dolichopodidae). An International Journal of Dipterological Research, 16(2): 133-146.

Negrobov, O.P. \& Satô, M. 2006. The comparative characteristic of fauna of family Dolichopodidae of the Japan and Far East of Russia. P. 178. In: 6th International Congress of Dipterology, Fukujoka, Japan, 2006. Fukujoka, Japan.

Negrobov, O.P., Selivanova, O.V. \& Maslova, O.O. 2016. A new species of the genus Dolichopus Latr. (Diptera, Dolichopodidae) from Sakhalin and Kuril Islands. Bulletin of Moscow Society of Naturalists, Biological Series, 121(5): 33-36. [In Russian]

Negrobov, O.P., Selivanova, O.V., Maslova, O.O. \& Chursina, M.A. 2013. Check-list of predatory flies of the family Dolichopodidae (Diptera) in the fauna of Russia. P. 47-93. In: I.Ya. Grichanov \& O.P. Negrobov (eds.). Fauna and taxonomy of Dolichopodidae (Diptera). Collection of papers. Saint Petersburg, VIZR RAAS Publ.

Negrobov, O.P. \& Shamshev, I.V. 1986. New species of the genus Sciapus Zeller (Dolichopodidae, Diptera) from Siberia. Trudy Zoologicheskogo instituta AN SSSR, 146: 17-22. [In Russian] 
Olson, D.M., Dinerstein, E., Wikramanayake, E.D., Burgess, N.D., Powell, G.V.N., Underwood, E.C., D'amico, J.A., Itoua, I., Strand, H.E., Morrison, J.C., Loucks, C.J., Allnutt, T.F., Ricketts, T.H., Kura, Y., Lamoreux, J.F., Wettengel, W.W., Hedao, P. \& Kassem, K.R. 2001. Terrestrial Ecoregions of the World: A New Map of Life on Earth: A new global map of terrestrial ecoregions provides an innovative tool for conserving biodiversity. BioScience, 51(11): 933-938. DOI: 10.1641/0006-3568(2001)051[0933:TEOTWA] 2.0.CO;2

Selivanova, O.V., Negrobov, O.P. \& Maslova, O.O. 2010. New data on fauna and morphology Dolichopus setimanus Smirnov (Dolichopodidae, Diptera). An International Journal of Dipterological Research, 21(3): 233-234.

Shamshev, I.V. 2004. A new species of the genus Microphorella Becker from the Far East of Russia, with notes on some morphological features in the Microphorinae (Diptera: Empidoidea). Studia Dipterologica, 2003, 10: 527-535.

Yang, D., Zhang, L.L. \& Zhang, K.Y. 2018. Species Catalogue of China, Volume 2, Animals, Insecta (VI), Diptera (2), Orthorrhaphous Brachycera. Beijing, Science Press. 387 pp.

(C) Far Eastern entomologist (Far East. entomol.) Journal published since October 1994. Editor-in-Chief: S.Yu. Storozhenko

Editorial Board: A.S. Lelej, S.A. Belokobylskij, M.G. Ponomarenko, V.A. Mutin, E.A. Beljaev, E.A. Makarchenko, A.V. Gorochov, T.M. Tiunova, M.Yu. Proshchalykin, S.A. Shabalin, V.M. Loktionov

Address: Federal Scientific Center of the East Asia Terrestrial Biodiversity (former Institute of Biology and Soil Science), Far East Branch of the Russian Academy of Sciences, 690022, Vladivostok-22, Russia.

E-mail: storozhenko@biosoil.ru web-site: http://www.biosoil.ru/fee 